\title{
One-stage operation for hydatid disease of lung and liver: Principles of treatment
}

\author{
İsmail Cüneyt Kurul, MD* \\ Salih Topcu, MD \\ Tamer Altinok, MD \\ Ulkü Yazici, MD \\ Irfan Tastepe, MD \\ Sadi Kaya, MD \\ Güven Cetin, MD
}

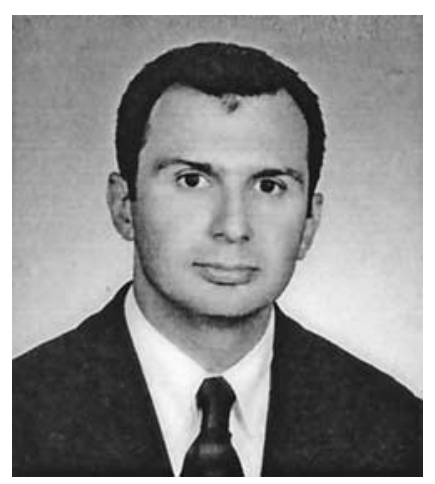

Dr Kurul

From the Department of Thoracic Surgery, Atatürk Center for Chest Disease and Thoracic Surgery, Ankara, Turkey.

Received for publication Feb 8, 2002; revisions requested March 28, 2002; revisions received May 1, 2002; accepted for publication June 4, 2002.

Address for reprints: Cüneyt Kurul, MD, Oyak Sitesi Blok 1/12, 06610 Çankaya, Ankara, Turkey (E-mail: ckurul@hotmail. com).

*Present address: Assistant Professor, Gazi University Medical Faculty, Department of Thoracic Surgery.

J Thorac Cardiovasc Surg 2002;124:1212-5

Copyright $\odot 2002$ by The American Association for Thoracic Surgery

0022-5223/2002 \$35.00+0 $\quad \mathbf{1 2 / 1 / 1 2 7 3 1 4}$

doi: $10.1067 / \mathrm{mtc} .2002 .127314$
Objective: Hydatid disease is endemic in many countries throughout the world. Although we do not have exact figures, the disease is prevalent in Turkey. A considerable number of patients have additional liver cysts as well. In this study we reviewed our experience with hydatid disease of the lung and liver and discussed the principles of treatment.

Methods: From 1971 to 1999,2509 patients with pulmonary hydatidosis were referred to us, and 485 of them had concomitant liver cysts. Of these, 405 patients had cysts located on the dome of the liver, and they were operated on with phrenotomy through a right thoracotomy. Eighty patients who were found to have concomitant liver cysts in the lower part of the liver were referred to general surgery for a laparotomy.

Results: Hydatid cysts located in the lungs were managed by means of cystotomy and capitonnage. For liver cysts, cystotomy and inversion of the cavity with sutures was the surgical method of choice, and a drain was left in place. In case of multiple cysts in the liver, needle aspiration was preferred. Twelve major complications, including excessive biliary drainage and bronchobiliary fistula, occurred in these patients.

Conclusions: We believe that management of pulmonary and hepatic cysts simultaneously through the thoracic route is convenient and should be encouraged in patients because this prevents a second operation. Needle aspiration can be applied only for liver cysts. It is absolutely contraindicated in lung hydatid cysts.

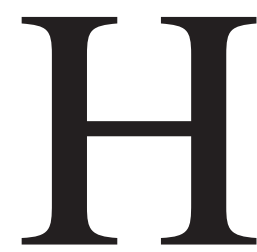

ydatid disease is a parasitosis and is endemic in many sheepraising regions throughout the world, especially in the Mediterranean countries. This disease is quite prevalent in Turkey. It is characterized by round lesions in the lungs and liver. A considerable number of patients with lung hydatid cysts also have liver cysts. ${ }^{1-4}$ Here we evaluate our patients with lung and liver cysts and present our experience. Also, we discuss the principles of treatment of hydatid disease.

\section{Patients and Methods}

From 1971 to 1999, 2509 patients underwent 2604 operations because of hydatid disease in our center. There were 1232 male and 1277 female patients whose ages ranged from 2 to 86 years, with a median age of 29.2 years. Concomitant liver cysts were found in 485 of them. In 405 of these patients, the cysts were located on the diaphragmatic surface of the liver, and phrenotomy through a right thoracotomy was the method of approach. The remaining 80 patients with cysts located in the lower part of the liver were referred to general surgery for 

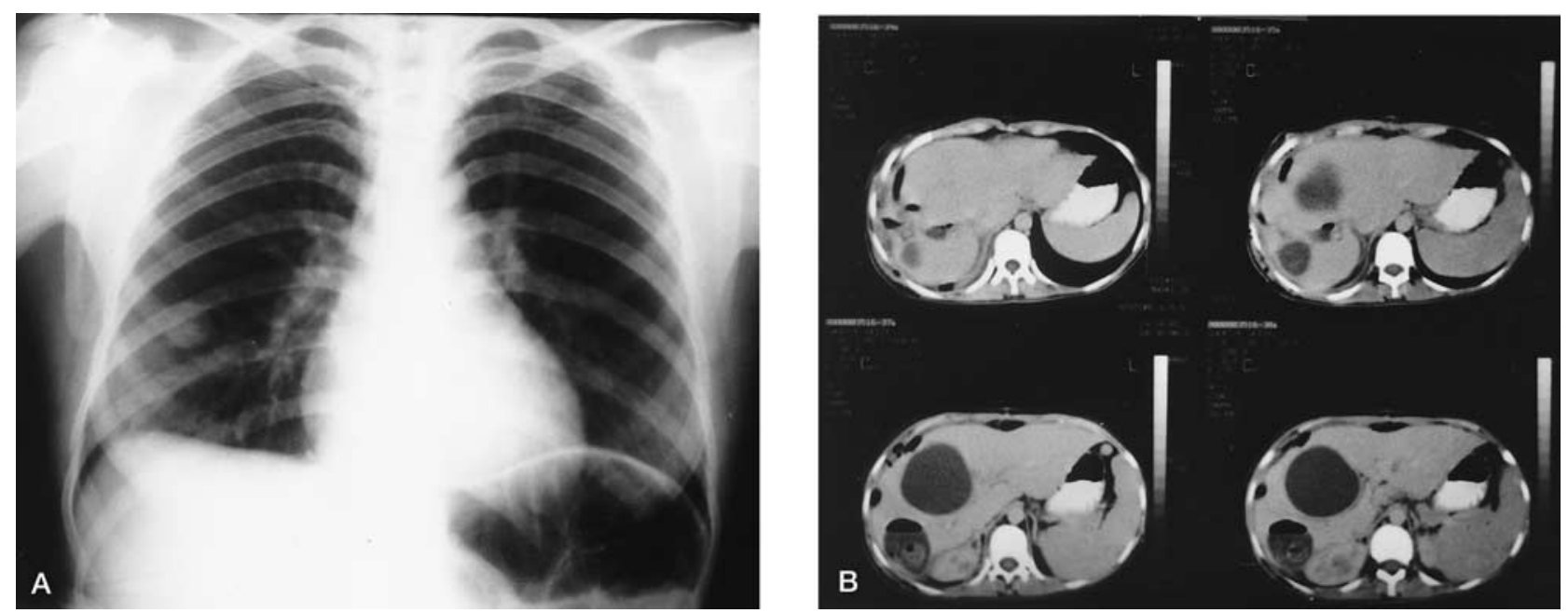

Figure 1. A, Chest $x$-ray film showing the elevation of the right leaf of the diaphragm. B, Computed tomographic scans of the same patient showing multiple hydatid cysts within the liver.

laparotomy. Of the 405 patients with concomitant lung and liver cysts with subphrenic location, 335 (82\%) had right lung, 14 (4\%) had left lung, and 56 (14\%) had bilateral lung cysts.

On admission, $60 \%$ of all the patients either had other parenchymatous lesions of the lung or pleural complications, including pneumothorax and pleural effusions. Empyema was found in 36 patients. In patients with empyema, the operation was delayed until the patient had recovered after the drainage of the empyema.

\section{Results}

In the simultaneous operation for lung and liver cysts, a posterolateral thoracotomy was done through the seventh intercostal space. The lung cysts were treated first. After posterolateral thoracotomy, the lung was freed from all adhesions to the chest wall. Then the edges of the wound and the surface of the lung other than the cyst surface was covered with sponges soaked in saline solution and diluted (20\%) povidone-iodine solution to prevent inadvertent implantation of scolices or a daughter cyst.

While the lungs were kept inflated, a large needle connected to the suction tip was inserted into the cyst. An antiscolicidal agent was not injected into the cystic cavity before needle aspiration. When the cyst was aspirated and its fluid evacuated, the most prominent part of the cyst was opened (cystotomy), and the cyst membrane was removed with ring forceps. Then the cavity was irrigated with saline solution and cleaned with sponges moistened with diluted povidone-iodine. The bronchial openings were sutured. The residual cavity starting from the deepest level, with a space of 1.5 to $2 \mathrm{~cm}$ left between each layer, was obliterated with absorbable purse-string sutures (polygalactin 910, Vicryl; Ethicon, Inc, Somerville, NJ; capitonnage), and then the diaphragm was incised radially above the palpated liver cyst. After the liver and the cyst surface had been freed from the adherent diaphragm, the contents of the cyst were aspirated with a large-bore needle. Cystotomy was performed to remove the germinative membrane. If there was no bile leak on the inner surface of the cyst, the cavity and the remaining pericystic liver tissue was inverted with sutures, and the subdiaphragmatic area was drained with a latex drain below the diaphragm. If bile drainage existed on the inner surface of the cyst, the biliary openings were sutured first, and the cavity was inverted. Then the subdiaphragmatic area was drained below the diaphragm with a latex drain. If there was combined biliary drainage and pus in the cavity, a latex drain was placed after the biliary openings had been sutured, and the cavity was inverted. The subdiaphragmatic area was drained also.

Generally, no matter how big the cysts are, we prefer not to put an external drain in the cystic cavity for the liver; the exception is in the case of suppuration. Only the subphrenic region is drained, and the drain is frequently removed on the third day. If there is a second cystic cavity or multiple cysts near or under the superficial cyst and if these cysts are detected as type I or type II with ultrasonographic examination, aspiration of the other cysts from the inner part of the first cyst is applied. This procedure has been named transcystic aspiration (Figure 1).

In the postoperative period, earlier in the study, mebendazole and later albendazole were used only for perforated cysts (10-20 mg/kg). The drug was given as 3 sequential, 28-day courses, with 14-day intervals between courses. The postoperative course in most of the patients was uneventful. Only a small number of complications occurred. Excessive biliary drainage occurred in 9 patients, and bronchobiliary fistula occurred in 3 patients. In these patients nasoduodenobiliary drainage was performed and had good 

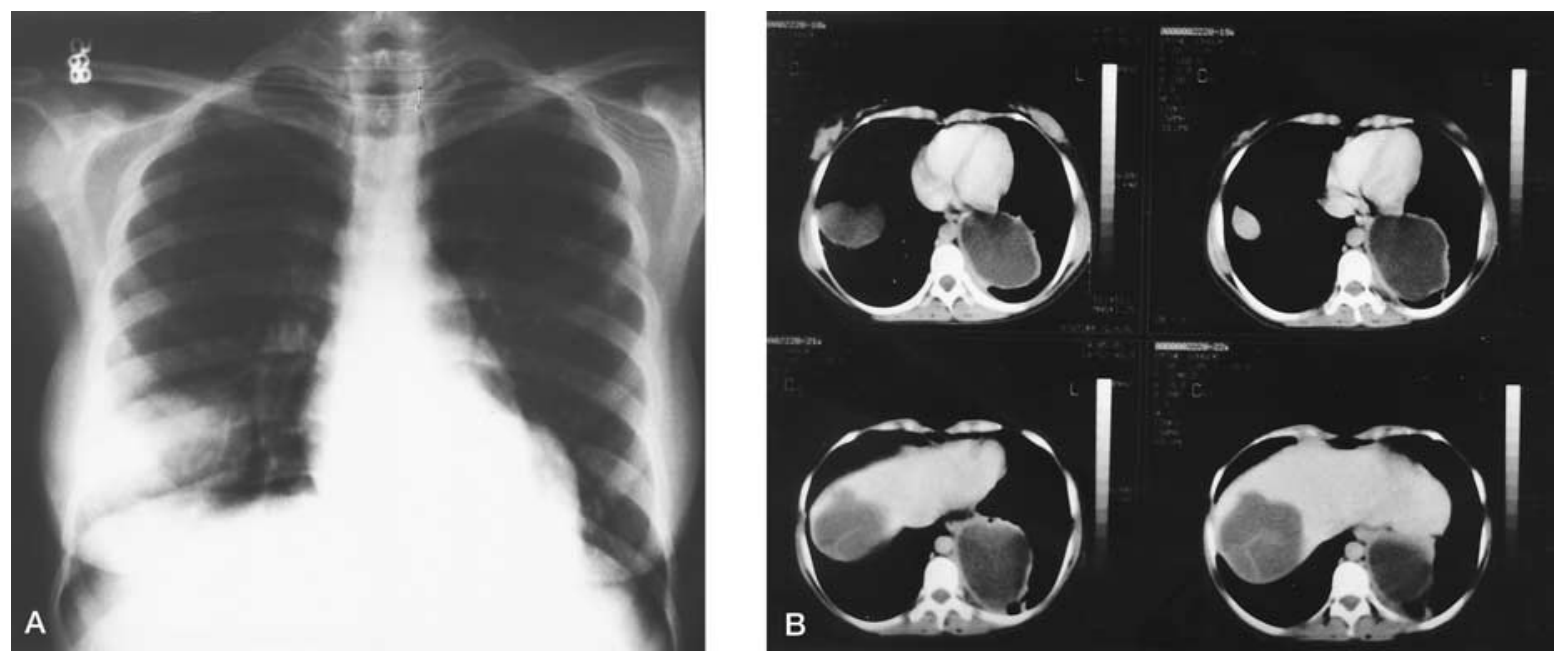

Figure 2. A, Bilateral cysts, one behind the heart and one on the right side. B, Computed tomographic scans of the same patient showing bilateral lung cysts and an additional right-sided liver cyst.

results. Tube drainage was also applied for patients with bronchobiliary fistula. From the early periods of this study, we have seen that for some patients who had uncomplicated liver cysts, external drainage has been applied. In these patients biliary drainage occurred 3 days later and lasted for a mean of 13 days. There were no perioperative deaths. All patients were subjected to a follow-up of 2 to 10 years. No recurrence was noticed for the cysts located in the liver. However, for lung hydatid cysts, recurrence of the disease was observed in 6 patients.

\section{Discussion}

Hydatid disease has been known since the time of Hippocrates. The clinical features of the disease have been described since then. Hydatidosis is a parasitic disease caused by the larval growth of the tapeworm (Echinococcus granulosus). The dog-sheep cycle is the paradigm for the life cycle of the parasite. One gets involved in this cycle by means of contact with infected dogs or consuming contaminated vegetables. ${ }^{1,2,4}$

Although the liver is the most common site of infection in adults, the most common site of infection in children is the lung. ${ }^{1-4}$ In addition, some patients might have hepatic cysts in addition to pulmonary cysts. Most of the cysts located in the liver might remain asymptomatic for a long time. With their enlargement, they might cause abdominal pain, discomfort, and a palpable mass (Figure 2). ${ }^{3,5}$ In cases of pulmonary cysts, patients might have dyspnea and nonproductive cough. Some patients might have blood-streaked sputum and thoracic pain. Vigorous coughing and expectoration of membranes are the symptoms of ruptured hydatid disease. ${ }^{1-3}$ Many hydatid cysts are asymptomatic and are found incidentally on chest radiographs. Plain chest radiographs are the major diagnostic tools in the diagnosis of hydatid disease. Computed tomographic imaging can show both intact and ruptured cysts. ${ }^{1-4}$ Ultrasonographic examination is another useful tool in the diagnosis of liver cysts. . $^{3-6}$ One who deals with a patient with lung hydatid cysts, especially those located on the right side, should keep in mind that a liver cyst must be searched for with ultrasonographic examination or computed tomography.

Surgical intervention is the main treatment for pulmonary and hepatic cysts. ${ }^{1-3,5}$ The principles of surgical treatment should be evacuation of the cyst with removal of the endocyst, avoidance of contamination, and management of the residual cavity, ${ }^{1-3}$ but the most important point in the operation is to preserve as much tissue as possible. For lung cysts, various surgical procedures have been described in the literature. ${ }^{1-3}$ These include conservative resections, such as cystotomy with or without capitonnage, or, rarely, radical resections, such as segmentectomy or lobectomy. The choice of surgical technique depends on the conditions encountered during the operation. In our clinic conservative surgical techniques, especially cystotomy and capitonnage of the residual cavity, are the methods of choice. Some might recommend leaving the cavity open, but we prefer to obliterate the cavity because of the risk of infection and abscess formation caused by a remaining cavity.

For the liver cysts located on the dome of the liver, we prefer the transthoracic approach: right thoracotomy and phrenotomy. In our experience with 405 patients, we have seen that even in giant liver cysts phrenotomy is convenient and has good results. For hepatic cysts, obliteration of the residual cavity without putting a drain causes good results. We have seen that external drainage causes a prolonged drainage in nonsuppurated cysts. Also, new biliary openings take form after external drainage. Suppuration is a very important factor in determining the treatment protocol. 3,5 
For suppurated liver cysts, we prefer to put a single rubber drain below the diaphragm into the cavity before inversion of the cavity.

For patients with bilateral hydatid disease, a median sternotomy approach can be applied, but as Çetin and colleagues ${ }^{7}$ reported, we did not prefer median sternotomy in patients with pleural complications and in patients with concomitant liver cysts because of the risk of mediastinitis and the risk of hepatobiliary fistula. ${ }^{1,7}$ Therefore, none of our patients with concomitant liver cysts underwent median sternotomy.

Although percutaneous needle aspiration is convenient in liver cysts, ${ }^{5}$ it is absolutely contraindicated in pulmonary cysts. ${ }^{1,2}$ Needle aspiration of lung cysts might cause complications, such as pneumothorax, anaphylactic reactions, and the development of new cysts. Patients will have the risk of superinfections in the following days. ${ }^{1,2}$ In our opinion transthoracic needle aspiration of suspected cysts must never be attempted.

Drugs have been used in the treatment of hydatid disease, but the results have been variable. We believe that medical treatment is of no value in pulmonary hydatid disease. However, various opinions concerning medical treatment exist. ${ }^{1-3}$ For lung hydatid disease, we do not recommend medical treatment in the preoperative period. During medical treatment, some complications, such as expectoration of membranes and anaphylactic shock, occur mostly as a result of perforations of the cysts. ${ }^{1,2}$ Medical treatment alone is not reliable in controlling the disease and is indicated only to prevent secondary recurrence in patients if there was a potential risk after the operation..$^{1-3}$

In conclusion, surgical intervention should be the primary treatment for hydatid disease. Liver cysts located subphrenically should be treated simultaneously with the lung hydatid disease. In patients with coexisting liver cysts, phrenotomy is more convenient and should be applied to prevent a second operation.

We thank Ergun Velidedeoglu, MD, for editing this article for language.

\section{References}

1. Topçu S, Kurul İC, Taştepe İ, Bozkurt D, Gülhan E, Çetin G. Surgical treatment of pulmonary hydatid cysts in children. J Thorac Cardiovasc Surg. 2000;120:1097-101.

2. Doğan R, Yüksel M, Çetin G, Süzer K, Alp M, Kaya S, et al. Surgical treatment of hydatid cysts of the lung: report on 1055 patients. Thorax. 1989;44:192-9.

3. Dhaliwal RS, Kalkat MS. One-stage surgical procedure for bilateral lung and liver hydatid cysts. Ann Thorac Surg. 1997;64:338-41.

4. Halilolu M, Saatçi I, Akhan O, Ozmen M, Besim A. Spectrum of imaging findings in pediatric hydatid disease. AJR Am J Roentgenol. 1997;169:1627-31.

5. Sayek, Yaln R, Sanaç Y. Surgical treatment of hydatid disease of the liver. Arch Surg. 1980;115:847-50.

6. Gharbi HA, Hassine W, Brauner MW, Dupuch K. Ultrasound examination of the hydatic liver. Radiology. 1981;139:459-63.

7. Çetin G, Doan R, Yüksel M, Alp M, Uçanak K, Kaya S, Ünlü M, Surgical treatment of bilateral hydatid disease of the lung via median sternotomy: experience in 60 consecutive patients. Thorac Cardiovasc Surg. 1988;36:114-7.

\section{Targeted}

The Journal of Thoracic and Cardiovascular Surgery gives you two tables of contents. The condensed table of contents tells you at a glance what topics and authors are presented each month. The expanded table of contents gives you a brief abstract of each article. You select only those articles of most interest to you for further reading. 